\title{
Metabolic acidosis status and mortality in patients on the end stage of renal disease
}

\author{
Vaia D. Raikou \\ The First Department of Medicine, Propaedaetic, National \& Kapodistrian University of Athens, \\ School of Medicine, Athens, Greece
}

\begin{abstract}
Background and Objectives: Uncorrected metabolic acidosis leads to higher death risk in dialysis patients. We observed the relationship between metabolic acidosis status and mortality rate in patients on renal replacement therapy during a median follow up time of 60 months. Methods: We studied 76 patients on an on-line hemodiafiltration. The dialysis adequacy was defined by $\mathrm{Kt} / \mathrm{V}$ for urea. The Framingham risk score (FRS) points were used to determine the 10-year risk for coronary heart disease. We examined the impact of high or low serum bicarbonate concentrations on mortality rate and on 10-year risk for coronary heart disease via the Kaplan-Meier method. Cox's model was used to evaluate a combination of prognostic variables, such as dialysis adequacy defined by $\mathrm{Kt} / \mathrm{V}$ for urea, age and serum bicarbonate concentrations. Results: We divided the enrolled patients in three groups according to serum bicarbonate concentrations ( $<20 \mathrm{mmol} / \mathrm{L}, 20-22 \mathrm{mmol} / \mathrm{L}$ and $>22 \mathrm{mmol} / \mathrm{L}$ ). Kaplan-Meier survival curve for the impact of serum bicarbonate concentrations on overall mortality was found significant (log-rank $=7.8, P=0.02$ ). The prevalence of serum bicarbonate less or more than $20 \mathrm{mmol} / \mathrm{L}$ on high FRS (> 20\%) by Kaplan-Meier curve was also found significant (log-rank $=4.9, P=0.02)$. Cox's model revealed the significant predictive effect of serum bicarbonate on overall mortality $(P=0.006, \mathrm{OR}=1.5,95 \% \mathrm{Cl}=1.12-1.98)$ in combination to $\mathrm{Kt} / \mathrm{V}$ for urea and age. Conclusion: Uncorrected severe metabolic acidosis, defined by serum bicarbonate concentrations less than $20 \mathrm{mmol} / \mathrm{L}$, is associated with a 10-year risk for coronary heart disease more than $20 \%$ and high overall mortality in patients on renal replacement therapy.
\end{abstract}

Key words: metabolic acidosis, dialysis, mortality, arterial stiffness, Framingham risk score

\section{INTRODUCTION}

Mortality is potentially elevated in patients with chronic kidney disease (CKD) in comparison to general population. Previously, it has been showed that patients in CKD stages 4 and 5 approached a rate of three-fold and six-fold higher mortality risk respectively, than patients with GFR $>60 \mathrm{~mL} / \mathrm{min} / 1.73 \mathrm{~m}^{2},{ }^{[1,2]}$ although dialysis mortality was shown as an eightfold higher age-standardised mortality as compared to the general population ${ }^{[3]}$.

The cardiovascular disease is recognized as the main reason for increased mortality among patients on renal replacement therapies, mainly due to the vascular occlusive disease defined by myocardial infarction, cerebrovascular strokes and ischemic events of the limbs. The underlying disease that leads to these events, progresses through various mechanisms including left ventricular hypertrophy, plaque formation, arterial stiffening and endothelial dysfunction. ${ }^{[4]}$

Although coronary atherosclerosis is a wellknown underlying cause of cardiovascular disease, arterial alterations include more widespread vascular changes that contribute to stiffening of arteries. Arterial hardening causes increased pulse wave velocity (PWV), increased pulse pressure (PP), cardiac overload and left ventricular hypertrophy. ${ }^{[5,6]}$ Large arterial PWV, which is a simple, noninvasive and reproducible marker, has been used in epidemiological studies during the last decades to quantify regional arterial stiffness demonstrating the predictive value 
of aortic stiffness for cardiovascular events in general population and in dialysis patients. ${ }^{[7]}$

Also, one of the most widely used 10-year risk assessment methods for the prediction of coronary heart disease is the Framingham risk score (FRS). According to the National Institutes of Health, the patients having chronic kidney disease are considered as having a coronary heart disease risk equivalent, meaning that they are primarily patients with a 10 -year risk for myocardial infarction or coronary death $>20 \%$, despite without known coronary heart disease. ${ }^{[8]}$

Metabolic acidosis, a common condition and an important manifestation of the late stage of chronic kidney disease, leads to clinically significant consequences, including bone disease disorders, protein energy wasting and a higher death risk. ${ }^{[9,10]}$ In spite of the risks related to uncorrected metabolic acidosis and the benefits with its treatment, a large rate of hemodialysis patients have suboptimal correction of metabolic acidosis. ${ }^{[1]}$

In this study, we observed the relationship between metabolic acidosis status and mortality rate in patients on renal replacement therapy during a median follow up time of 60 months.

\section{MATERIALS AND METHODS}

\section{Patient characteristics}

We included 76 patients in an on-line predilution hemodiafiltration. This is a dual-centre observational longitudinal retrospective study, examining the effect of metabolic acidosis status on overall mortality. The data collection was carried out during a median time of 60 months (from January-2007 to December-2011).

The study was reviewed and approved by the 'Laiko, University General Hospital of Athens' and the Renal Unit of 'Diagnostic and Therapeutic Centre of Athens Hygeia SA' Institutional Review Board. All study participants, or their legal guardians, provided informed written consent prior to study enrolment.

In total, 47 men and 29 women participated in our study, mean age being $62.2 \pm 15$ years old. In our data, 11 patients were initiated on the dialysis treatment after the initiation of this study and 65 patients were already in permanent dialysis therapy. Patients' contribution to the follow up ended at the time of death, renal transplantation, renal recovery, or at the past follow up (December 2011) (Figure 1).

We excluded the patients less than 18 years of age at the initiation of dialysis and the patients that had less than 6 months of follow-up. Patients without regular vascular hemodialysis access and who had dialysis catheter and those with autoimmune diseases, infections or malignancy were excluded from our study. The hemodialysis treatment was performed 3-times weekly with a dialysis time of 3.5-4 hours per session, a filter of $1.5-2 \mathrm{~m}^{2}$ surface area by high-flux synthetic membrane, defined by an ultrafiltration coefficient $>20 \mathrm{~mL} / \mathrm{h}^{[12]}$ and a blood flow of $350-400 \mathrm{~mL} / \mathrm{min}$. A bicarbonate-based ultrapure buffer dialysis solution was used with a dialysate flow rate of $500-600 \mathrm{~mL} / \mathrm{min}$, a calcium concentration of $1.50-1.75 \mathrm{mmol} / \mathrm{L}$, a sodium concentration of 138 $145 \mathrm{mmol} / \mathrm{L}$ and low molecular weight heparin as anticoagulant therapy. The final concentration of bicarbonate in dialysate was $32 \mathrm{mEq} / \mathrm{L}$. The dialysis dose defined by Kt/V/day for urea, which was calculated according to the formula of Daugirdas. ${ }^{[13]}$ The patients were excluded if they had Kt/V for urea less than 1.2. The enrolled patients had a good status and they did not have interdialytic peripheral oedema, high blood pressure, interdialytic orthostatic hypotension or other characteristics of an inaccurate dry body weight.

Cardiovascular disease was represented by the presence of coronary disease (CD, $n=25,32.9 \%$ ), which might be the underlying disease for heart failure or peripheral arterial disease. At the beginning of the study, the coronary syndrome was documented by the history of myocardial

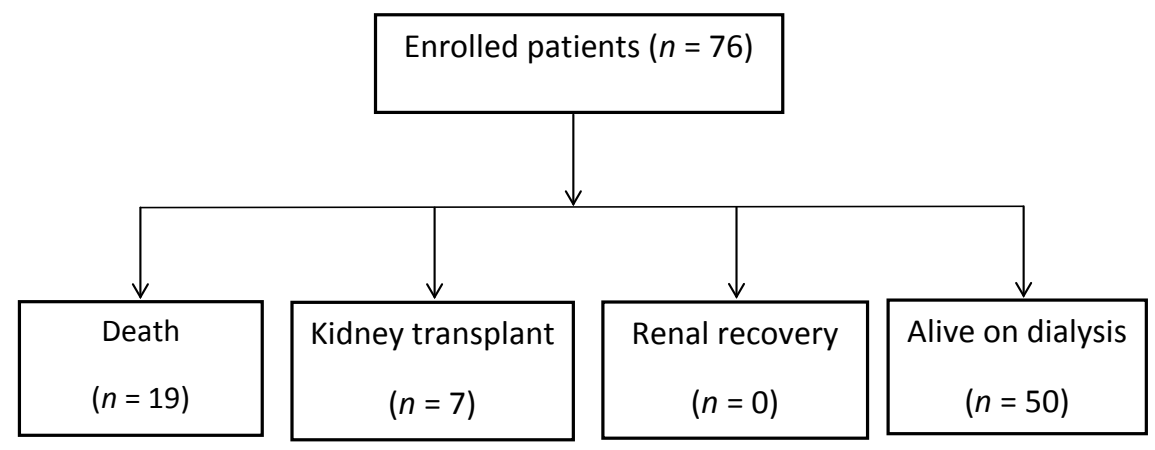

Figure 1: Study chart. The chart describes the participants' clinical outcomes. 
infarction, coronary artery angioplasty or bypass surgery, clinical signs of angina pectoris or electrocardiographic ischaemic findings. Also, the first and the current cardiovascular events during the study were written down as one event for the coronary artery disease manifestation.

Twenty-nine of the enrolled patients (a ratio of 38.2\%) were receiving anti-hypertensive drugs, such as calcium channel blockers, beta-blockers or inhibitors of angiotensin II receptors. Another receiving medication included statin, and only calcium-free phosphate binders were prescribed. All the studied patients were on erythropoetin-a or- $\beta$ therapy.

Nineteen of the studied patients were current smokers (a ratio of $25 \%$ ). 20 studied patients disposed urine volume up to $100 \mathrm{~mL} /$ day. For each included subject in present study, a separate Framingham risk score (FRS) sheet was used to determine the 10-year risk for coronary heart disease, according to the updated version of score points associated with the age, sex, total cholesterol, HDL, smoking status, systolic blood pressure and hypertension, either treated or untreated, excluding diabetes mellitus ${ }^{[8]} 37$ of the enrolled patients had an FRS less than 10\% (a ratio 48.7\%), 21 patients had an FRS equal to 10-20\% (a ratio 27.6\%) and 18 patients had an FRS more than 20\% (a ratio 23.7\%).

In our data, the renal failure was caused by hypertensive nephrosclerosis at a ratio of $32.9 \%$, chronic glomerulonephritis at a ratio of $28.9 \%$, polycystic disease at a ratio $11.8 \%$, diabetic nephropathy at a ratio equal to $9.2 \%$ and other causes at a ratio $17.1 \%$.

\section{Blood collection}

Blood samples were obtained just before the start of the mean weekly dialysis session in a twelve hours fasting state from the vascular access. In the end of the treatment, the blood pump speed was reduced to less than $80 \mathrm{~mL} / \mathrm{min}$ and blood samples were obtained at 2 min post-dialysis from the arterial dialysis tubing for the calculation of the adequacy of dialysis by Kt/V for urea. The samples were centrifuged immediately, and the serum was separated and processed for various assays.

In each subject, three sequences of samples (every month within 3 months) were received for the serum bicarbonate measurements, and their average was used for statistical analysis. The low serum bicarbonate level was considered in combination to low arterial $\mathrm{pH}$ (acidemia) and decreased PCO2.

\section{Laboratory measurements}

Albumin, high density lipoproteins (HDL) and low density lipoproteins (LDL) were measured by biochemical analysis and the ratio of LDL/HDL was calculated. Hematocrit and hemoglobin values were also measured. High sensitivity C-reactive protein (hsCRP) serum concentrations were measured using the enzyme linked immunosorbent assays (ELISA, immundiagnostik AG, Germany) according to the manufacturer's specifications. The concentrations of intact-parathormone (i-PTH) were measured by radioimmunoassay (CIS bio international/France). The serum bicarbonate concentrations were measured in gas machine (Roche, combas b 121) taking care of the blood specimens. ${ }^{[14]}$ The normalized protein catabolic rate for dry body mass (nPCR) was calculated from the urea generation rate. ${ }^{[15]}$ The body mass index (BMI) was obtained from height and post-dialysis body weight.

\section{Haemodynamic measurements}

Predialysis peripheral systolic and diastolic blood pressures (SBP and DBP respectively) in enrolled patients were calculated as the mean of 10 measurements during a treatment month using an automatic sphygmomanometer OMRON M4-I (Co. Ltd. Kyoto Japan). Mean peripheral pre-dialysis $\mathrm{BP}(\mathrm{MBP})$ was calculated as: $\mathrm{MBP}=\mathrm{DBP}+$ $1 / 3$ (SBP - DBP).

Electrocardiographical analysis and M-mode echocardiography were performed the day after dialysis with a Hewlett Packard SONOS 2500 using a $2.25 \mathrm{MHz}$ transducer to estimate the ejection fraction and the ischaemic findings according to the recommendations of the American Society of Echocardiography. ${ }^{[16]}$

Arterial stiffness was measured as carotid-femoral pulse wave velocity (c-f PWV) and carotid augmentation index (AIx) using the SphygmoCor system ${ }^{\circledR}$ (AtCor Medical Pty. Ltd, Sydney, Australia). In each subject, two sequences of measurements were performed, and their mean was used for statistical analysis. We recorded the carotid-femoral PWV by positioning one sensor over the right femoral artery and a second sensor over the left carotid artery. The distance between the two sensors was measured with a measuring tape, and three recordings of both pulse waveforms were performed (8-10 heart beats for each recording). The Complior software automatically detected the foot of each pulse waveform from the two arterial sites and then measured the mean distance between the two feet as being the travel time of the wave. PWV was then computed using the formula: $\mathrm{PWV}=$ travel distance/travel time, as previously validated. ${ }^{[17]}$

Central systolic blood pressure (SBP), diastolic blood pressure (DBP), mean blood pressure (MBP), PP, and the time of return of the reflected wave $(\mathrm{Tr})$ were derived. Pressure and time of first peak (P1 and T1) and second peak (P2 and T2), and central augmented pressure (AP) were obtained. Central augmentation index (AIx) was 
computed $(\mathrm{AP}=\mathrm{P} 2-\mathrm{P} 1 ; \mathrm{AIx}=(\mathrm{AP} / \mathrm{PP}) \times 100)$ and corrected for a heart rate of 75 beats/minute.

\section{Statistical analysis}

Univariate analyses were conducted to describe the distributions of baseline variables. We used SPSS 15.0 statistical package for Windows (SPSS Inc, Chicago, Illinois) for all statistical analyses. Data were expressed as mean \pm standard deviation or as median value (interquartile range) for that which showed skewed distribution; Differences between mean values were assessed by using one-way ANOVA tests for three groups and unpaired $t$-test for two groups, and the data that showed the skewed distributions were compared with Mann-Whitney $U$ - test. Significance statements referred to $\mathrm{P}$ values of two-tailed tests that were less than 0.05 . Chi-square analysis was used for the correlation between categorical variables.

Survival curves and graphical assessment of proportional hazards were based on the Kaplan-Meier method. We examined the impact of high or low serum bicarbonate concentrations on mortality rate via the Kaplan-Meier method. Because of the follow-up time not being the same for all the enrolled patients, we also used KaplanMeier curves to investigate the relationship between FRS groups of patients and both, high or low serum bicarbonate levels and high or low hsCRP values. Cox's model was used to evaluate the interactions of prognostic variables in a multivariate manner, such as dialysis adequacy defined by $\mathrm{Kt} / \mathrm{V}$ for urea and age in combination with serum bicarbonate concentrations.

\section{RESULTS}

The characteristics of the studied population at the time of inclusion are listed in Table 1.

We divided the enrolled study patients into three groups according to the serum bicarbonate concentrations (SBC). The group 1 included patients with SBC less than $20 \mathrm{mmol} / \mathrm{L}(n=33)$, the group 2 had patients with SBC equal to $20-22 \mathrm{mmol} / \mathrm{L}(n=34)$ and the group 3 included patients with SBC more than $22 \mathrm{mmol} / \mathrm{L}(n=9)$.

Kaplan-Meier analysis for the impact of SBC on overall mortality for the study patients divided into three groups was found significant (log-rank $=7.8, P=0.02$ ) (Figure 2). The prevalence of SBC, less or more than $20 \mathrm{mmol} / \mathrm{L}$ on high FRS (group of patients with FRS $>20 \%$ ) by Kaplan-Meier curve, was also found significant (log-rank $=4.9, P=0.02)$ (Figure 3). Kaplan-Meier analysis showed a significant impact of less or more than the mean value of hsCRP equal to $7.97 \pm 5.8 \mathrm{mg} / \mathrm{L}$ on high FRS $(>20 \%)$ (log-rank $=3.7, P=0.04)$.

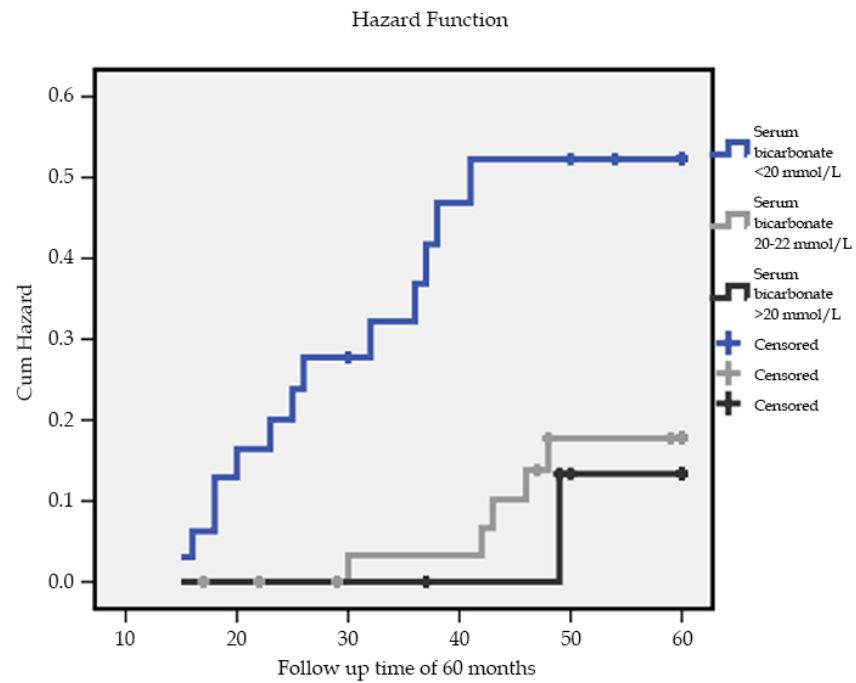

Figure 2: Kaplan-Meier survival curve for the impact of serum bicarbonate concentrations $(\mathrm{SBC})$ on death hazard function for 76 dialysis patients divided in three groups during a median follow up time of 60 months (log-rank $=7.8, P=$ 0.02). The group 1 included patients with $\mathrm{SBC}$ less than $20 \mathrm{mmol} / \mathrm{L}(n=33)$, the group 2 had patients with SBC equal to $20-22 \mathrm{mmol} / \mathrm{L}(n=34)$ and the group 3 included patients with $\mathrm{SBC}$ more than $22 \mathrm{mmol} / \mathrm{L}(n=9)$.

\begin{tabular}{ll}
\hline Table 1: Baseline characteristics & \\
\hline Parameters & $n=76$ \\
\hline Male & $47(61.8)$ \\
Age (years) & $62.2 \pm 15.01$ \\
Dialysis vintage (years) & $5(3-10)$ \\
BMI (kg/m²) & $24.4 \pm 3.03$ \\
Kt/ V for urea & $1.29(1.25-1.49)$ \\
nPCR (g/kg/day) & $2.3 \pm 0.5$ \\
SBC (mEq/L) & $20.09 \pm 2.05$ \\
LDL/HDL & $2.33 \pm 0.86$ \\
i-PTH (pg/mL) & $126.5(63.3-233.0)$ \\
hsCRP (mg/L) & $7.97 \pm 5.8$ \\
Albumin (g/dL) & $4.0(3.8-4.2)$ \\
MBP (mmHg) & $98.4 \pm 12.6$ \\
C-f PWV (m/s) & $11.3 \pm 1.83$ \\
Alx (\%) & $24.1 \pm 2.1$ \\
PP (mmHg ) & $58.22 \pm 19.3$ \\
FRS (\%) & $11.6 \pm 9.3$ \\
Hemoglobin (g/dL) & $11.8 \pm 1.3$ \\
Coronary disease & $25(32.9)$ \\
hypertension & $29(38.2)$ \\
Current smoking & $19(25)$ \\
Diabetes mellitus & $7(9.2)$ \\
\hline
\end{tabular}

Results are means $\pm \mathrm{SD}, n(\%)$ or median $\left(25^{\text {th }}-75^{\text {th }}\right.$ percentiles $) . \mathrm{BMI}$ : body mass index, nPCR: normalized protein catabolic rate, SBC: serum bicarbonate concentrations, HDL: high density lipoproteins, LDL: low density lipoproteins, i-PTH: intact-parathormone, PP: pulse pressure, MBP: mean peripheral pre-dialysis, Framingham risk score: FRS, Alx: augmentation index, c-f PWV: carotid-femoral pulse wave velocity. 
Cox's model revealed significant predictive effect of SBC on overall mortality $(P=0.006, \mathrm{OR}=1.5,95 \% \mathrm{CI}=1.12$ $1.98)$ in combination with the dialysis adequacy defined by $\mathrm{Kt} / \mathrm{V}$ for urea and age.

In Table 2, the differences between the groups of patients according to SBC are shown. We observed that the group 1 of patients (SBC $<20 \mathrm{mmol} / \mathrm{L}$ ) had higher FRS, i-PTH, PP, age, hsCRP, c-fPWV and AIx, but lower albumin than the group 3 of patients (SBC $>22 \mathrm{mmol} / \mathrm{L})$. The differences between the groups 1 and 2 and between 2 and 3 were similar, noting statistically significant difference for c-fPWV and AIx between the three groups of patients.

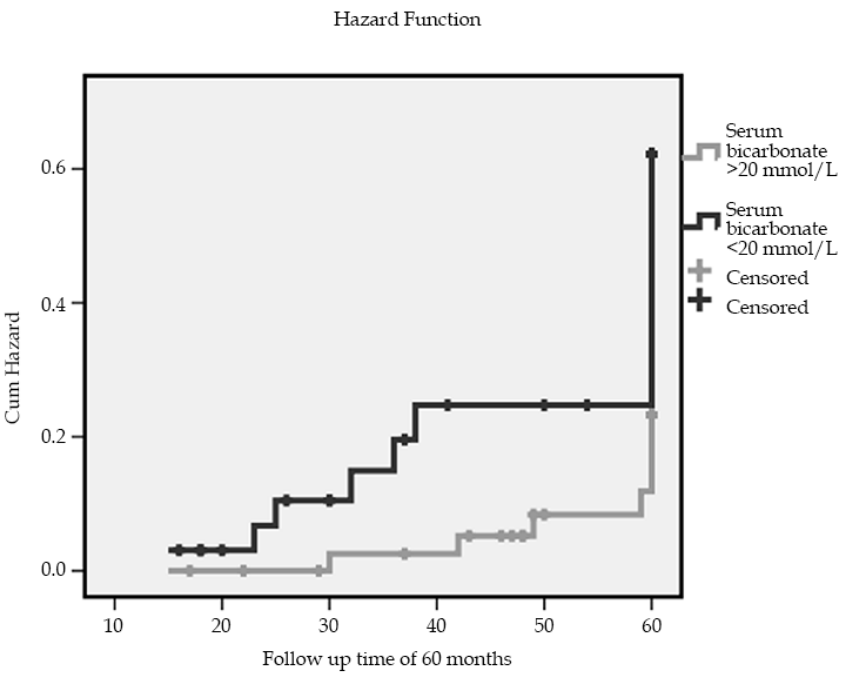

Figure 3: Kaplan-Meier survival curve for the prevalence of serum bicarbonate concentrations less or more than $20 \mathrm{mmol} / \mathrm{L}$ on high 10 -year risk for coronary heart disease by Framingham risk score (FRS $>20 \%$ ) during a median follow up time of 60 months (log-rank $=4.9, P=0.02$ ).
Chi-Square analysis showed significant association between $\mathrm{SBC}$ and FRS groups of patients $\left(\chi^{2}=4.3, P=0.03\right)$ (Figure 4).

Multivariable correlations showed significant inverse relationship between serum bicarbonate concentrations and hsCRP, c-fPWV (Figure 5) and AIx $(r=-0.281, P=0.01 ; r$ $=-0.642, P=0.001$; and $r=-0.517, P=0.001$, respectively).

\section{DISCUSSION}

It has been already established that the end-stage renal disease (ESRD) results in accelerated atherosclerosis and increased morbidity and mortality. ${ }^{[4]}$ Even when the

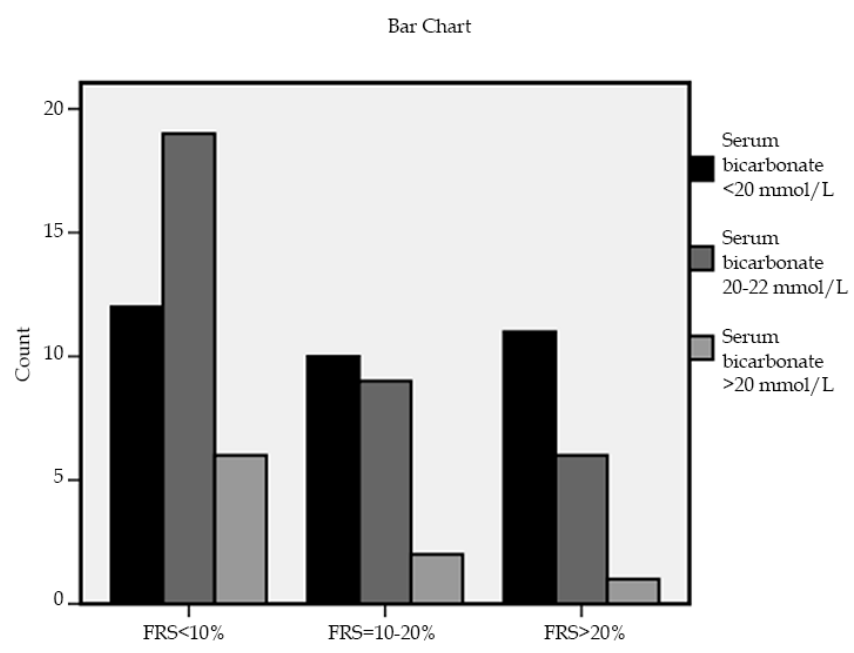

Figure 4: association between the three groups of patients according to serum bicarbonate concentrations and according to Framingham risk score points $\left(\chi^{2}\right.$ $=4.3, P=0.03)$.

\begin{tabular}{|c|c|c|c|}
\hline & $\begin{array}{l}\text { SBC }<20 \mathrm{mmol} / \mathrm{L} \\
n=33\end{array}$ & $\begin{array}{l}\mathrm{SBC}=20-22 \mathrm{mmol} / \mathrm{L} \\
n=34\end{array}$ & $\begin{array}{l}\mathrm{SBC}>22 \mathrm{mmol} / \mathrm{L} \\
n=9\end{array}$ \\
\hline Age (years) & $64.6 \pm 14.3$ & $64.5 \pm 13.1$ & $44.8 \pm 13.9^{*}$ \\
\hline BMI $\left(\mathrm{kg} / \mathrm{m}^{2}\right)$ & $24.7 \pm 3.4$ & $24.1 \pm 2.8$ & $24.4 \pm 1.76$ \\
\hline $\mathrm{Kt} / \mathrm{V}$ for urea & $1.39 \pm 0.2$ & $1.38 \pm 0.19$ & $1.36 \pm 0.18$ \\
\hline nPCR (g/kg/day) & $2.26 \pm 0.6$ & $2.45 \pm 0.45$ & $2.57 \pm 0.35$ \\
\hline $\mathrm{SBC}(\mathrm{mmol} / \mathrm{L})$ & $18.1 \pm 1.27$ & $21.1 \pm 0.6$ & $23.07 \pm 1.0^{*}$ \\
\hline LDL/HDL & $2.17 \pm 0.84$ & $2.38 \pm 0.79$ & $2.7 \pm 1.14$ \\
\hline i-PTH (pg/mL) & $191.9 \pm 210.8$ & $186.4 \pm 187.6$ & $116.04 \pm 84.6$ \\
\hline hsCRP (mg/L) & $9.01 \pm 5.4$ & $7.77 \pm 6.07$ & $4.96 \pm 5.7^{*}$ \\
\hline Albumin $(\mathrm{g} / \mathrm{dL})$ & $3.94 \pm 0.3$ & $3.95 \pm 0.5$ & $4.11 \pm 0.22$ \\
\hline $\mathrm{MBP}(\mathrm{mmHg})$ & $96.5 \pm 12.5$ & $100.25 \pm 13.5$ & $98.7 \pm 9.6$ \\
\hline$c-f$ PWV (m/s) & $12.3 \pm 1.6$ & $10.9 \pm 1.5^{*}$ & $9.3 \pm 1.04 *$ \\
\hline Alx $(\%)$ & $25.2 \pm 2.1$ & $23.7 \pm 1.98 *$ & $22.4 \pm 1.3^{*}$ \\
\hline $\mathrm{PP}(\mathrm{mmHg})$ & $60.45 \pm 21.3$ & $57.9 \pm 19.01$ & $51.1 \pm 10.2$ \\
\hline Hemoglobin (g/dL) & $11.8 \pm 1.58$ & $11.7 \pm 0.96$ & $12.5 \pm 1.5$ \\
\hline FRS (\%) & $13.5 \pm 9.6$ & $11.2 \pm 9.05$ & $6.4 \pm 7.6^{*}$ \\
\hline
\end{tabular}

${ }^{*} P<0.05$. BMI: body mass index, nPCR: normalized protein catabolic rate, SBC: serum bicarbonate concentrations, HDL: high density lipoproteins, LDL: low density lipoproteins, i-PTH: intact-parathormone, PP: pulse pressure, MBP: mean peripheral pre-dialysis, Framingham risk score: FRS, Alx: augmentation index, c-f PWV: carotid-femoral pulse wave velocity. 
patients are undergoing renal replacement therapy, the mortality remains high, mainly for cardiovascular causes due either to uremia-related risk factors (such as anemia, hyperparathyroidism, inflammation, oxidative stress and malnutrition) ${ }^{[18,19]}$ or to the traditional ones (age, male gender, diabetes, obesity, hypertension, smoking, dyslipidemia). ${ }^{[20]}$ The cardiovascular parameters are not completely corrected by dialysis therapy.

Metabolic acidosis is an important characteristic particularly in advanced renal disease. This is the result of a decreased ability to excrete non-volatile acid and the reduced renal synthesis of bicarbonate. The maintenance dialysis therapies are often not able to completely correct the base deficit. Acidemia is believed to be an important cause of adverse consequences and it is associated with an increased relative risk for death in dialysis patients. ${ }^{[9,21,22]}$

In agreement to the above, in our data also we found significant impact of the serum bicarbonate concentrations, which was divided into three levels, on the overall mortality during our median follow up time of 60 months. Also, the serum bicarbonate concentrations were independent predictors for mortality rate after the adjustment for dialysis adequacy and age.

Previously, it had been suggested that the correction of metabolic acidosis results in reduction of mortality and hospitalization in hemodialysis and peritoneal dialysis patients, thus this is one of the goals of effective dialysis. ${ }^{[2,24]}$ Also, the previous studies in animals and in humans reported that correction of metabolic acidosis was shown to attenuate the rate of decline in renal function. ${ }^{[25]}$

Regarding the optimal correction of metabolic acidosis, previously it has been reported that the therapeutic targets for serum bicarbonate may differ by dialysis modality. ${ }^{[6,27]}$ However, a recent study conducted on a large sample of 121, 351 patients at the last stage of renal disease showed that despite the measured serum bicarbonate being significantly higher in peritoneal dialysis patients than in intermittent hemodialysis, a level of serum bicarbonate more than $22 \mathrm{mEq} / \mathrm{L}$ for all ERSD patients was most beneficial for survival, irrespectively of dialysis modality. ${ }^{[28]}$

In the present data, we studied our subjects after a division of serum bicarbonate concentrations into three levels - less than $20 \mathrm{mmol} / \mathrm{L}, 20-22 \mathrm{mmol} / \mathrm{L}$ and more than $22 \mathrm{mmol} / \mathrm{L}$. We observed that the patients with serum bicarbonate less than $20 \mathrm{mmol} / \mathrm{L}$ had significantly higher FRS, age, hsCRP, c-fPWV and AIx than the patients with serum bicarbonate more than $22 \mathrm{mmol} / \mathrm{L}$. Also, they had higher i-PTH and PP, but lower Hb and serum albumin in comparison to the patients with serum bicarbonate

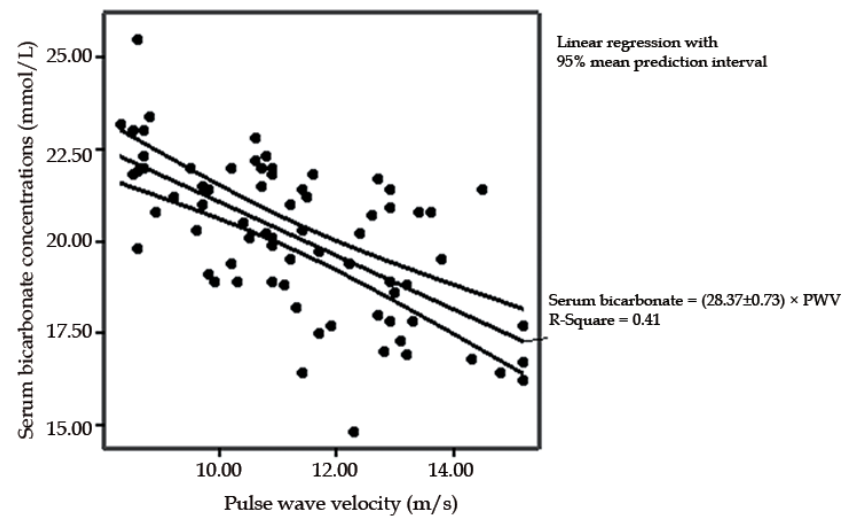

Figure 5: Significantly inverse association between c-fPWV and serum bicarbonate concentrations ( $r=-0.642, P=0.001)$.

more than $22 \mathrm{mmol} / \mathrm{L}$. These differences were smaller in comparison to the patients with serum bicarbonate less than $20 \mathrm{mmol} / \mathrm{L}$ and the patients with serum bicarbonate 20-22 mmoll between them. However, c-fPWV and AIx differed significantly between all groups of patients.

Supporting this, we found a significantly inverse correlation between serum bicarbonate and both, c-fPWV, AIx and hsCRP serum concentrations.

Increased pulse wave velocity and augmentation index are integrated indexes of vascular function and structure. They estimate the arterial stiffness, which is a strong predictor of cardiovascular mortality in general population and in dialysis patients. ${ }^{[29]}$ Also, the elevated PP is another consequence of arterial stiffening and vascular calcification. The positive relationship between the extent of vascular calcification and arterial stiffness has been shown previously, and it may explain the increased cardiovascular events seen in dialysis patients. ${ }^{[30]}$

On the other hand, as already reported, the role of metabolic acidosis on vascular calcification and bone disease, as the mineral metabolism disturbances act through the existing metabolic acidosis in dialysis patients. ${ }^{[31]}$ Also, acidosis promotes inflammation of the arterial wall, releasing cytokines that may induce vascular calcification. ${ }^{[32]}$ Malnutrition, another common condition defined by low serum albumin and a risk factor for poor outcome in renal disease, may be related to acidemia due to an increased protein catabolism, decreased protein synthesis, endocrine abnormalities and inflammation among patients with renal disease. ${ }^{[9,33]}$

Additionally, in our data, we observed significant prevalence of serum bicarbonate lower or higher than $20 \mathrm{mmol} / \mathrm{L}$ on high 10-year risk for coronary heart disease (FRS > $20 \%$ ) during our median follow up time of 60 months. Supportively, the relationship between the three groups of 
patients according to the serum bicarbonate concentrations and according to the 10-year risk for coronary heart disease by FRS was found statistically significant.

These findings support that metabolic acidosis results in detrimental effects and patients with low bicarbonate level should be treated properly even though they are receiving dialysis therapy, since uncorrected acidosis may be one of the reason for the incomplete correction of cardiovascular parameters by dialysis therapy.

In the meantime, we noted significant impact of high hsCRP serum concentrations on high 10-year risk for coronary heart disease (FRS $>20 \%$ ) during our median follow up time of 60 months. Indeed, the relationship between inflammation, atherosclerosis and cardiovascular disease has been established. ${ }^{[19]}$

According to our results, the underlying pathophysiological mechanisms for both, increased 10-year risk for coronary heart disease and elevated mortality in dialysis patients with low serum bicarbonate levels, mainly less than 20 $\mathrm{mmol} / \mathrm{L}$, include metabolic disorders, malnutrition, bone disease, chronic inflammation and arterial stiffness, which is definitively a powerful predictor of mortality in patients on dialysis.

In conclusion, uncorrected severe metabolic acidosis, defined by serum bicarbonate concentrations less than $20 \mathrm{mmol} / \mathrm{L}$, is associated with 10-year risk for coronary heart disease more than $20 \%$ and high overall mortality in patients on renal replacement therapy. The main pathophysiological mechanisms may be the accelerated arterial stiffness, malnutrition and inflammation connected to an environment of advanced acidemia.

\section{Conflict of Interest}

None declared.

\section{Financial Support}

None.

\section{REFERENCES}

1. Neovius M, Jacobson SH, Eriksson JK, Elinder CG, Hylander B. Mortality in chronic kidney disease and renal replacement therapy: a populationbased cohort study. BMJ Open 2014;4:e004251.

2. Coresh J, Turin TC, Matsushita K, Sang Y, Ballew SH, Appel LJ, et al. Decline in estimated glomerular filtration rate and subsequent risk of end-stage renal disease and mortality. JAMA 2014;311:2518-31.

3. de Jager DJ, Grootendorst DC, Jager KJ, van Dijk PC, Tomas LM, Ansell $\mathrm{D}$, et al. Cardiovascular and noncardiovascular mortality among patients starting dialysis. JAMA 2009;302:1782-9.

4. Inaba M, Ueda M. [Vascular Calcification - Pathological Mechanism and
Clinical Application]. The significance of arterial calcification in unstable plaques]. Clin Calcium 2015;25:679-86.

5. Rodriguez RA, Shea B, Hae R, Burns KD. The impact of intervention strategies that target arterial stiffness in end-stage renal disease: a systematic review protocol. Syst Rev 2016;5:118.

6. Yerram P, Chaudhary K. Calcific uremic arteriolopathy in end stage renal disease: pathophysiology and management. Ochsner J 2014;14:380-5.

7. Ma Y, Zhou L, Dong J, Zhang X, Yan S. Arterial stiffness and increased cardiovascular risk in chronic kidney disease. Int Urol Nephrol 2015;47:1157-64.

8. D’Agostino RB Sr, Vasan RS, Pencina MJ, Wolf PA, Cobain M, Massaro JM, et al. General Cardiovascular Risk Profile for Use in Primary Care The Framingham Heart Study. Circulation 2008;117:743-53.

9. Yenigun EC, Aypak C, Turgut D, Aydin MZ, Dede F. Effect of metabolic acidosis on QT intervals in patients with chronic kidney disease. Int J Artif Organs 2016;39:272-6.

10. Yamamoto T, Shoji S, Yamakawa T, Wada A, Suzuki K, Iseki K, et al. Predialysis and Postdialysis $\mathrm{pH}$ and Bicarbonate and Risk of All-Cause and Cardiovascular Mortality in Long-term Hemodialysis Patients. Am J Kidney Dis 2015;66:469-78.

11. Chiu YW, Mehrotra R. What should define optimal correction of metabolic acidosis in chronic kidney disease? Semin Dial 2010;23:411-4.

12. Susantitaphong P, Siribamrungwong M, Jaber BL. Convective therapies versus low-flux hemodialysis for chronic kidney failure: a meta-analysis of randomized controlled trials. Nephrol Dial Transplant 2013;28:285974.

13. Sternby J, Daugirdas JT. Theoretical basis for and improvement of Daugirdas' second generation formula for single-pool Kt/V. Int J Artif Organs 2015;38:632-7.

14. Kirschbaum B. Spurious metabolic acidosis in hemodialysis patients. Am J Kidney Dis 2000;35:1068-71.

15. Luman M, Jerotskaja J, Lauri K, Fridolin I. Dialysis dose and nutrition assessment by optical on-line dialysis adequacy monitor. Clin Nephrol 2009;72:303-11.

16. Han SS, Cho GY, Park YS, Baek SH, Ahn SY, Kim S, et al. Predictive value of echocardiographic parameters for clinical events in patients starting hemodialysis. J Korean Med Sci 2015;30:44-53.

17. Zhang Y, Agnoletti D, Xu Y, Wang JG, Blacher J, Safar ME. Carotidfemoral pulse wave velocity in the elderly. J Hypertens 2014;32:1572-6.

18. Liu M, Li XC, Lu L, Cao Y, Sun RR, Chen S, Zhang PY. Cardiovascular disease and its relationship with chronic kidney disease. Eur Rev Med Pharmacol Sci 2014;18:2918-26.

19. Peev V, Nayer A, Contreras G. Dyslipidemia, malnutrition, inflammation, cardiovascular disease and mortality in chronic kidney disease. Curr Opin Lipidol 2014;25:54-60.

20. Wen CP, Cheng TY, Tsai MK, Chang YC, Chan HT, Tsai SP, et al. All-cause mortality attributable to chronic kidney disease: A prospective cohort study based on 462293 adults in Taiwan. Lancet 2008;371:2173-82.

21. Kalantar-Zadeh K. Moderator's view: Higher serum bicarbonate in dialysis patients is protective. Nephrol Dial Transplant 2016;31:1231-4.

22. Dobre M, Yang W, Chen J, Drawz P, Hamm LL, Horwitz E, et al. Association of serum bicarbonate with risk of renal and cardiovascular outcomes in CKD: a report from the Chronic Renal Insufficiency Cohort (CRIC) study. Am J Kidney Dis 2013;62:670-8.

23. Kato A, Kido R, Onishi Y, Kurita N, Fukagawa M, Akizawa T, et al. Association of serum bicarbonate with bone fractures in hemodialysis patients: the mineral and bone disorder outcomes study for Japanese CKD stage 5D patients (MBD-5D). Nephron Clin Pract 2014;128:79-87.

24. Chang TI, Oh HJ, Kang EW, Yoo TH, Shin SK, Kang SW, et al. A low serum bicarbonate concentration as a risk factor for mortality in peritoneal dialysis patients. PLoS One 2013;8:e82912.

25. de Brito-Ashurst I, Varagunam M, Raftery MJ, Yaqoob MM. Bicarbonate supplementation slows progression of CKD and improves nutritional status. J AmSoc Nephrol 2009;20:2075-84. 
26. Davies S. Clinical Practice Guidelines Module 3b: Peritoneal Dialysis, 2008. Available at: www.renal.org. Accessedon April 9, 2012.

27. Mactier R. Clinical Practice Guidelines: Hemodialysis, 2008. Available at: www.renal.org. Accessed on April 9, 2012.

28. Vashistha T, Kalantar-Zadeh K, Molnar MZ, Torle K, Mehrotra R. Dialysis Modality and Correction of Uremic Metabolic Acidosis: Relationship with All-Cause and Cause-Specific Mortality. Clin J Am Soc Nephrol 2013;8:254-64.

29. Fortier C, Agharazii M. Arterial Stiffness Gradient. Pulse (Basel) 2016;3:159-66.

30. Breznik S, Ekart R, Hren M, Rupreht M, Balon BP. Radiographic assessment of vascular calcification, aortic pulse wave velocity, ankle-brachial index and fibroblast growth factor- 23 in chronic hemodialysis patients. Ther Apher Dial 2013;17:378-83.
31. Oka M, Ohtake T, Mochida Y, Ishioka K, Maesato K, Moriya H, et al Correlation of coronary artery calcification with pre-hemodialysis bicarbonate levels in patients on hemodialysis. Ther Apher Dial 2012;16:26771.

32. Ori Y, Bergman M, Bessler H, Zingerman B, Levy-Drummer RS, Gafter U, et al. Cytokine secretion and markers of inflammation in relation to acidosis among chronic hemodialysis patients. Blood Purif 2013;35:181-6.

33. de Oliveira CM, Vidal CL, Cristino EF, Pinheiro FM Jr, Kubrusly M. Metabolic acidosis and its association with nutritional status in hemodialysis. J Bras Nefrol 2015;37:458-66.

How to cite this article: Raikou VD. Metabolic acidosis status and mortality in patients on the end stage of renal disease. J Transl Intern Med 2016; 4: 170-77. 